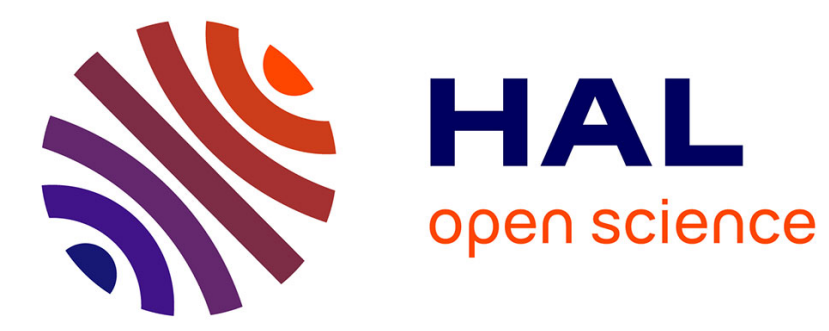

\title{
Pseudo-Gaussian cylindrical acoustical beam - Axial scattering and radiation force on an elastic cylinder
}

\author{
F.G. Mitri, Z.E.A. Fellah, G.T. Silva
}

\section{To cite this version:}

F.G. Mitri, Z.E.A. Fellah, G.T. Silva. Pseudo-Gaussian cylindrical acoustical beam - Axial scattering and radiation force on an elastic cylinder. Journal of Sound and Vibration, 2014, 333 (26), pp.7326 7332. hal-01082365

\section{HAL Id: hal-01082365 https://hal.science/hal-01082365}

Submitted on 2 Dec 2014

HAL is a multi-disciplinary open access archive for the deposit and dissemination of scientific research documents, whether they are published or not. The documents may come from teaching and research institutions in France or abroad, or from public or private research centers.
L'archive ouverte pluridisciplinaire HAL, est destinée au dépôt et à la diffusion de documents scientifiques de niveau recherche, publiés ou non, émanant des établissements d'enseignement et de recherche français ou étrangers, des laboratoires publics ou privés. 


\title{
Pseudo-Gaussian Cylindrical Acoustical Beam - Axial Scattering and Radiation Force on an Elastic Cylinder
}

\author{
F.G. Mitri ${ }^{\text {a, }}$, Z.E.A. Fellah ${ }^{\text {b }}$, G.T. Silva ${ }^{c}$
}

${ }^{\mathrm{a} C h e v r o n, ~ A r e a ~} 52$ Technology - ETC, 5 Bisbee Ct., Santa Fe, NM, 87508, United States.

bLMA, CNRS, UPR 7051, Aix-Marseille University, Centrale Marseille, F-13402 Marseille Cedex 20, France.

${ }^{\mathrm{c}}$ Physical Acoustics Group, Instituto de Física, Universidade Federal de Alagoas, Maceió, AL 57072900, Brasil.

*Corresponding author: F.G. Mitri (e-mail: F.G.Mitri@ieee.org). 
Abstract - Making use of the addition theorem for the cylindrical wave functions and the complexsource-point method in cylindrical coordinates, an exact solution to the Helmholtz equation is derived, which corresponds to a tightly focused (or collimated) cylindrical quasi-Gaussian beam with arbitrary waist. The solution is termed "quasi-Gaussian" to make a distinction from the standard Gaussian beam solution obtained in the paraxial approximation. The advantage of introducing this new solution is the efficient and fast computational modeling of tightly focused or quasi-collimated cylindrical wave-fronts depending on the dimensionless waist parameter $k w_{0}$, where $k$ is the wave number of the acoustical radiation. Moreover, a closed-form partial-wave series expansion is obtained for the incident field, which has the property that the axial scattering (i.e. along the direction of wave propagation) and the axial acoustic radiation force (which is a time-averaged quantity) on a cylinder, can be calculated without any approximations in the limit of linear acoustical waves in a nonviscous fluid. Examples are found where the extinction in the radiation force function plot is found to be correlated with conditions giving reduction of the backscattering from an elastic cylinder. Those results are useful in beam-forming design, particle manipulation in acoustic tweezers operating with focused cylindrical beams, and the prediction of the scattering and radiation forces on a cylindrical particle or liquid bridges.

Keywords: addition theorem, complex-source-point method, quasi-Gaussian beams, scattering, radiation force, elastic cylinder. 


\section{Introduction}

Acoustic beam modeling of focused ultrasonic transducers is an important process in beam-forming design in order to optimize design parameters, such as geometry, focus depth, acoustic beam width and directivity. This process consists of determining the acoustic pressure and its spatial distribution in front of the radiating surface. Various software packages exist (such as Abersim [1], Field II [2], FOCUS [3] or k-Wave [4]), in which the acoustic beam-profile can be modeled using the Rayleigh-Sommerfeld diffraction integral [5]. Apertures ranging from simple geometrical shapes, such as planar, spherically/cylindrically focused waves, to more complex (arbitrarily-shaped) wave-fronts have been considered in the aforementioned packages. However, the numerical simulations for the beam-forming are generally time-consuming, which require numerical integration procedures. It is therefore important to devise fast and efficient analytical tools which allow optimized design of experimental tools and the development of novel devices.

Along that line of research, the complex-source-point method (CSPM) $[6,7,8,9,10]$ has been introduced, and exact solutions of the Helmholtz equation were developed and analyzed for the fast beam-profile modeling of spherically focused quasi-Gaussian beams, both in acoustics [11] and optics [12]. Moreover, the CSPM has been applied for cylindrically focused quasi-Gaussian optical beams [13]. The "quasi-Gaussian" terminology has been used to make a distinction from the standard Gaussian beam solution obtained in the paraxial approximation, which does not satisfy the Helmholtz equation.

The purpose of this investigation is to extend the analysis in [13] to include cylindrical acoustical beams, thus, providing an efficient tool in the modeling of strongly focused and quasi-collimated scalar cylindrical pressure fields. Cylindrically symmetric wave-fields originate in the wave diffraction theory as an exact solution of the Helmholtz equation (pp. 75-77 [14]). Moreover, the analysis is extended to derive an exact partial-wave series expansion, which is used to predict the axial (i.e. along the direction 
of wave propagation) scattering and radiation force on an elastic cylinder centered on the beam's focus.

\section{Method}

Following Eq.(6) in [13], the scalar velocity potential field of a cylindrical quasi-Gaussian beam propagating in fluid medium of density $\rho_{0}$ and speed of sound $c_{0}$ with normal incidence (i.e. $\alpha=0$, where $\alpha$ is the tilt angle of the incident wavefront with respect to the axis of the cylinder), can be expressed as,

$$
\Phi_{q G}^{c y l .}=\frac{\Phi_{0}}{I_{0}\left(k x_{R}\right)} J_{0}\left(k \rho^{-}\right) e^{-i \omega t}
$$

where $\Phi_{0}$ is the characteristic velocity potential amplitude at the center of the coordinate system, $J_{0}($.$) is$ the cylindrical Bessel function of the first kind [15], $I_{0}($.$) is the modified cylindrical Bessel function of$ the first kind, $k$ is the wave number, $\rho^{-}=\sqrt{\left(x-i x_{R}\right)^{2}+y^{2}}$, and $\theta^{-}=\cos ^{-1}\left[\left(x-i x_{R}\right) / \rho^{-}\right]$(Fig. 1). At normal incidence, $\alpha=0$, there is no propagation along the axis of the cylinder such that the axial wave number $k_{z}=k \sin \alpha=0$. The parameter $x_{R}=k w_{0}^{2} / 2$ (known as the Rayleigh range) is expressed through a variable $w_{0}$, which corresponds to the beam's waist. Note that the incident pressure field can be obtained from the velocity potential such that $p=i \omega \rho_{0} \Phi_{q G}^{c y l .}$.

To illustrate the solution presented in Eq.(1), the spatial distribution of the velocity potential magnitude is numerically evaluated in the cross-sectional plane for different values of the dimensionless waist $k w_{0}$. The results are displayed in Fig. 2. As expected, at low $k w_{0}$ values, the cylindrical beam is tightly focused (or strongly divergent), and begins to spread as $k w_{0}$ increases. This behavior was also observed for spherical quasi-Gaussian waves (Fig. 1 in [16]).

Consider now a cylindrical particle centered on the axis of the cylindrical coordinates system. Making 
use of the expression for the addition theorem for the cylindrical wave functions (Eq.(2) on p. 360 in Ref. [17]) taking into account the geometry of the present problem (Fig. 1), the expression of the velocity potential field given in Eq.(1), is rewritten in term of a partial-wave expansion as,

$$
\begin{aligned}
\Phi_{q G}^{c y l .} & =\frac{\Phi_{0} e^{-i \omega t}}{I_{0}\left(k x_{R}\right)} \sum_{n=-\infty}^{+\infty} J_{n}(k r) J_{n}\left(-i k x_{R}\right) e^{i n(\pi-\theta)}, \\
& =\Phi_{0} e^{-i \omega t} \sum_{n=-\infty}^{+\infty} \Lambda_{n}\left(k x_{R}\right) i^{n} J_{n}(k r) e^{-i n \theta},
\end{aligned}
$$

where $\theta=\cos ^{-1}(x / r)$, and

$$
\Lambda_{n}\left(k x_{R}\right)=I_{n}\left(k x_{R}\right) / I_{0}\left(k x_{R}\right)
$$

It is instructive to analyze the dependence of the velocity potential field of a cylindrical quasiGaussian beam on the coefficient $\Lambda_{n}\left(k x_{R}\right)$ as given by Eq.(3). In the limit of tightly focused (or strongly divergent) beams (i.e. $k w_{0}<1$ ), with the assumption that $k x_{R} \ll \sqrt{n+1}$, the asymptotic form of the modified cylindrical Bessel function of the first kind is used [15]; $\left.I_{n}\left(k x_{R}\right)\right|_{k_{R} \rightarrow 0} \rightarrow\left(k^{2} w_{0}^{2} / 4\right)^{n} / n !$. In this limit, it can be verified from Eq.(3) that $\left.\Lambda_{0}\left(k x_{R}\right)\right|_{k_{R} \rightarrow 0}=1,\left.\quad \Lambda_{1}\left(k x_{R}\right)\right|_{k_{R} \rightarrow 0} \leq 1 / 4$, and $\left.\Lambda_{n \geq 2}\left(k x_{R}\right)\right|_{k x_{R} \rightarrow 0} \rightarrow 0$, so the series in Eq.(2) will only contain the monopole $(n=0)$ and dipole $(|n|=1)$ partial-waves.

For collimated beams (i.e. $k w_{0} \gg>1$ ), using the asymptotic expansion of the modified cylindrical Bessel function of the first kind [15]; $\left.I_{n}\left(k x_{R}\right)\right|_{k x_{R} \rightarrow \infty} \rightarrow e^{k x_{R}} / \sqrt{2 \pi k x_{R}}$, the coefficient $\Lambda_{n}\left(k x_{R}\right)$ in Eq.(3) approaches unity. Such properties for tightly focused (or strongly divergent) or quasi-collimated beams can be further analyzed by numerical computation of Eq.(3) versus the partial-wave number $n$. The results are displayed in Fig. 3 for various $k w_{0}$ values. Indeed, as $k w_{0}$ increases and tends to infinity, the coefficient $\Lambda_{n}\left(k x_{R}\right)=1$, and Eq.(2) reduces to the series expansion of plane waves. 
The partial-wave series expansion for the incident cylindrical quasi-Gaussian beam Eq.(2) allows the evaluation of the axial acoustical scattering (i.e. along the direction of wave propagation) by a cylinder with its center coinciding with the origin of the cylindrical coordinates system. An elastic polymer material is chosen here to illustrate the analysis, however, the formulation remains valid for fluid $[18,19,20]$, viscoelastic $[21,22,23,24]$, layered cylinders [25,26], shells [27,28,29], and layered cylindrical shells $[30,31]$.

Consider that the elastic cylinder of radius $a$ is immersed in non-viscous water. The scattered velocity potential field by the cylinder produces a cylindrical wave-field expressed as,

$$
\Phi_{s, q G}^{c y l .}=\Phi_{0} e^{-i \omega t} \sum_{n=-\infty}^{+\infty} \Lambda_{n}\left(k x_{R}\right) i^{n} C_{n} H_{n}^{(1)}(k r) e^{-i n \theta}
$$

where $H_{n}^{(1)}($.$) is the cylindrical Hankel function of the first kind of order n$, and $C_{n}$ are the scattering coefficients determined by applying appropriate boundary conditions [32] at the interface fluid-structure, with the assumption that the surrounding fluid is nonviscous. These functions depend on the cylinder's elastic parameters such as the longitudinal, the shear or transverse sound speed and the mass densities of both the fluid and the cylinder. After arithmetic manipulation, it is found that these coefficients are equivalent to those obtained from the study of the acoustic scattering by plane waves (See Eq.(4) in [33]).

In the far-field $k r \rightarrow \infty$, the asymptotic form for the Hankel function is $\left.H_{n}^{(1)}(k r)\right|_{k r \rightarrow \infty} \rightarrow \sqrt{(2 / \pi k r)} e^{i(k r-n \pi / 2-\pi / 4)}$, so that a steady-state far-field form-function can be defined as $[34,35]$

$$
\begin{aligned}
f_{\infty}(k a, \theta) & =\sqrt{\frac{2 r}{a}}\left(\frac{\Phi_{s, q G}^{c y l}}{\Phi_{0}}\right) e^{-i(k r-\omega t)} \\
& =\frac{2}{\sqrt{i \pi k a}} \sum_{n=-\infty}^{+\infty} \Lambda_{n}\left(k x_{R}\right) C_{n} e^{-i n \theta}
\end{aligned}
$$


Now that the expressions for the incident and scattered fields are known, the evaluation of the acoustic radiation force (ARF) becomes possible. Taking advantage of the far-field scattering, the ARF is expressed as [36],

$$
\langle\mathbf{F}\rangle \underset{k r \rightarrow \infty}{=} \frac{1}{2} \rho_{0} k^{2} \int_{0}^{2 \pi} \operatorname{Re}\left\{\Phi_{i s}\right\} \mathrm{d} \mathbf{S}
$$

where,

$$
\Phi_{i s}=\left[\left(\frac{i}{k} \frac{\partial \Phi_{q G}^{c y l}}{\partial r}-\Phi_{q G}^{c y l .}\right) \Phi_{s, q G}^{c y l . *}-\left|\Phi_{s, q G}^{c y l .}\right|^{2}\right]
$$

and the cylinder's differential surface is $\mathrm{d} \mathbf{S}=\operatorname{Lrd} \theta \mathbf{e}_{\mathbf{r}}$ with a length $L$. The unit vector in the radial direction is $\mathbf{e}_{\mathbf{r}}$ and the symbol $\langle\cdot\rangle$ denotes time-averaging.

Taking the asymptotic limits for Eq.(2) and Eq.(4) in the far-field scattering limit (i.e. $k r \rightarrow \infty$ ), and substituting them into Eq.(6) using Eq.(7) as well as the property of the following angular integral,

$$
\int_{0}^{2 \pi} e^{i\left(n^{\prime}-n\right) \theta} \cos \theta d \theta=\pi\left(\delta_{n, n+1}+\delta_{n, n-1}\right)
$$

where $\delta_{i j}$ is the Kronecker delta function, the axial radiation force can be expressed as,

$$
\begin{aligned}
F_{x} & =\langle\mathbf{F}\rangle \cdot \mathbf{e}_{\mathbf{r}} \\
& =Y_{q G} S E_{0},
\end{aligned}
$$

where $S=2 a L$ is the cross-sectional surface of the cylinder, $E_{0}=\frac{1}{2} \rho_{0} k^{2}\left|\Phi_{0}\right|^{2}$ is a characteristic energy density, and $Y_{q G}$ is the radiation force function, which is the radiation force per unit cross-sectional surface and unit energy density. Its expression is given by,

$$
Y_{q G}=-\frac{1}{I_{0}^{2}\left(k x_{R}\right) k a} \sum_{n=-\infty}^{+\infty} I_{n}\left(k x_{R}\right)\left[\begin{array}{l}
I_{n+1}\left(k x_{R}\right)\left(\beta_{n} \beta_{n+1}+\alpha_{n} \alpha_{n+1}+\alpha_{n+1}\right) \\
+I_{n-1}\left(k x_{R}\right)\left(\beta_{n} \beta_{n-1}+\alpha_{n} \alpha_{n-1}+\alpha_{n-1}\right)
\end{array}\right]
$$

where $\alpha_{n}=\operatorname{Re}\left\{C_{n}\right\}$, and $\beta_{n}=\operatorname{Im}\left\{C_{n}\right\}$. 
Note that in the limit of collimated beams (i.e. $k w_{0} \gg>1$ ), Eq.(10) can be further simplified using the asymptotic limit for the modified cylindrical Bessel function of the first kind, and the expression for the radiation force function for plane progressive waves is recovered, and is given by,

$$
Y_{p}=-\frac{1}{k a} \sum_{n=-\infty}^{+\infty}\left[\left(1+\alpha_{n}\right)\left(\alpha_{n+1}+\alpha_{n-1}\right)+\beta_{n}\left(\beta_{n+1}+\beta_{n-1}\right)\right]
$$

\section{Numerical results and discussion}

The following examples are now considered to illustrate the analysis by plotting the magnitude of the

backscattering form-function $\left|f_{\infty}(k a, \theta=\pi)\right|$ as well as the radiation force function $Y_{q G}$ for a cylindrical quasi-Gaussian beam incident upon a polymethylmetacrylate $\left(\right.$ PMMA) elastic cylinder $\left(\rho_{P M M A}=1191\right.$ $\left.\mathrm{kg} / \mathrm{m}^{3} ; c_{L}=2690 \mathrm{~m} / \mathrm{s} ; c_{T}=1340 \mathrm{~m} / \mathrm{s}\right)$ immersed in water $\left(\rho_{0}=1000 \mathrm{~kg} / \mathrm{m}^{3} ; c_{0}=1500 \mathrm{~m} / \mathrm{s}\right)$. The computations are evaluated in the dimensionless frequency range $0<k a \leq 10$ for two values of the dimensionless beam waist parameter $k w_{0}=1$, corresponding to a tightly focused (or strongly divergent) cylindrical beam, and $k w_{0}=25$, corresponding to a quasi-collimated beam. It is important to emphasize that the diffraction limit is reached for a beam waist $w_{0}$ approaching half the wavelength $\lambda / 2$ of the incident radiation (i.e., $k w_{0}=\pi$ ) [37]. Beams with a dimensionless waist $k w_{0}<\pi$ are not directional, and below this limit, wave reflections at the aperture of the focused source generate exponentially decaying evanescent waves which become relatively important as $k w_{0} \rightarrow 0$ [38]. If one aims to construct a beam with a dimensionless waist $k w_{0}<\pi$ using a finite source confined in a bounded space, it becomes essential to consider the effects of the evanescent wavefield at the focus. Note here that the corresponding source terms contributing to that part of the evanescent component become exponentially large at the source relative to the homogeneous (propagating) part of the wavefield. This fact may introduce a significant complication in modeling the tightly focused field as exponentially-large source 
amplitudes have to be taken into consideration so as to retain the evanescent component at the focus of the beam to make a tight focus, and the linear assumptions of the Helmholtz equation do not hold. In the present study, the focus is on propagating waves and the theoretical development disregards the evanescent contributions to the homogeneous field.

In Fig. 4, the numerical plots for $\left|f_{\infty}(k a, \theta=\pi)\right|$ are displayed for $k w_{0}=1$ (solid line) and $k w_{0}=25$ (dashed line). An interesting observation is related to the plot for $k w_{0}=1$ (i.e. solid line) in which a reduction in the backscattering is noticed. Inspection of Fig. 5 shows that the reduction in the backscattering has a major effect on the radiation force function $Y_{q G}$ that approaches zero at those particular values of $k a$ (Fig. 5); that is, the reduction in the backscattering shown in the plots for $\left|f_{\infty}(k a, \theta=\pi)\right|$ closely matches the zeros of $Y_{q G_{0}}$ for $k w_{0}=1$. This behavior has been also observed in the study of the backscattering and radiation force of a spherical quasi-Gaussian beam on an elastic sphere (Fig. 2 in [39]), but was not observed in the case of a rigid sphere [40]. At the specific $k a$ values for which the backscattering is reduced, the radiation force function $Y_{q G} \rightarrow 0$ for $k w_{0}=1$, and the transmission of acoustical waves through the elastic cylinder in the forward direction (i.e. axial direction $\theta=0$ ) is maximized. This is not the case as $k w_{0}$ increases to reach 25 (i.e. dashed curves) so the plots for $\left|f_{\infty}(k a, \theta=\pi)\right|$ and $Y_{q G}$ approach the plane wave limit, respectively (Fig. 1 in [23] \& Fig. 1 in [24]).

It is noticeable from Fig. 5 that for a tightly focused (or strongly divergent) cylindrical quasi-Gaussian beam centered on the elastic cylinder, the (axial) radiation force function approaches closely to zero, however, is not found to be negative (i.e. not a force of attraction). In some situations, theoretical predictions have demonstrated the existence of a negative (pulling) force on a rigid cylinder in a focused Gaussian beam [41] (and spheres as well [42,43]), however, the mathematical expression for the incident Gaussian beam provided therein differs from Eq.(2). Note also that in the context of Bessel 
beams, earlier works have shown the existence of a force of attraction on a sphere $[44,45,46]$, which is associated with a reduction in the backscattering as well [47].

It is also important to note an equivalent partial-wave series expansion for the incident field Eq.(2). Using the properties of the cylindrical Bessel and modified Bessel functions of the first kind $i^{n} J_{n}(z)=i^{-n} J_{-n}(z)$, and $I_{-n}(z)=I_{n}(z)$, the series in Eq.(2) can be expressed as,

$$
\begin{aligned}
\Phi_{q G}^{c y l .} & =\Phi_{0} e^{-i \omega t} \sum_{n=-\infty}^{+\infty} \Lambda_{n}\left(k x_{R}\right) i^{n} J_{n}(k r) e^{-i n \theta} \\
& =\Phi_{0} e^{-i \omega t} \sum_{n=0}^{+\infty} \Lambda_{n}\left(k x_{R}\right) i^{n} \varepsilon_{n} J_{n}(k r) \cos (n \theta),
\end{aligned}
$$

where $\varepsilon_{n}$ is the Neumann factor, defined by $\varepsilon_{0}=1$, and $\varepsilon_{j}=2$, for $j=1, \ldots n$.

In a real source coordinate system such that $x_{0}=0$, a closely related generalized partial-wave series expansion that resembles to Eq.(12) has been provided (Eq.(1) in [48]), which can be applied for computing the axial acoustic radiation force in the cases of plane travelling waves [24,25,36,49], plane standing [26,29,50] or quasi-standing waves [33], diverging cylindrical waves [51], and diverging or converging cylindrical travelling, standing and quasi-standing waves $[48,52]$. Following the generalized formalism (Eq.(15) in [48]), and noting that $\Lambda_{n}\left(k x_{R}\right)$ as given by Eq.(3) is a real coefficient, the radiation force function for a cylindrical quasi-Gaussian beam $Y_{q G}$, is expressed as,

$$
\begin{aligned}
Y_{q G} & =-\frac{2}{k a} \sum_{n=0}^{\infty}\left\{\Lambda_{n}\left(k x_{R}\right) \Lambda_{n+1}\left(k x_{R}\right)\left[\alpha_{n}+\alpha_{n+1}+2 \alpha_{n} \alpha_{n+1}+2 \beta_{n} \beta_{n+1}\right]\right\} \\
& =-\frac{2}{I_{0}^{2}\left(k x_{R}\right) k a} \sum_{n=0}^{\infty}\left\{I_{n}\left(k x_{R}\right) I_{n+1}\left(k x_{R}\right)\left[\alpha_{n}+\alpha_{n+1}+2 \alpha_{n} \alpha_{n+1}+2 \beta_{n} \beta_{n+1}\right]\right\},
\end{aligned}
$$

which is equivalent to Eq.(10).

It is obvious from Eq.(13) that for a collimated beam $\left(k w_{0} \rightarrow \infty\right)$, the expression for the radiation force function reduces to the plane wave result [49], which is equivalent to Eq.(11). 


\section{Conclusion}

In summary, an exact acoustical beam solution that satisfies the wave equation is introduced and some of its intrinsic characteristics, as well as its scattering and radiation force properties are delineated for a tightly focused and quasi-collimated cylindrical wave-fronts incident upon an elastic cylinder. Those results are useful in beam-forming design as well as modeling the interaction of a cylindrical quasiGaussian acoustical beam with a cylindrical particle for applications in acoustical tweezers, imaging and particle manipulation. 


\section{REFERENCES}

[1] T. Varslot, G. Taraldsen, Computer simulation of forward wave propagation in soft tissue, IEEE Transactions on Ultrasonics, Ferroelectrics and Frequency Control 52 (2005) 1473-1482.

[2] J.A. Jensen, N.B. Svendsen, Calculation of pressure fields from arbitrarily shaped, apodized, and excited ultrasound transducers, IEEE Transactions on Ultrasonics, Ferroelectrics and Frequency Control 39 (1992) 262-267.

[3] R.J. McGough, Rapid calculations of time-harmonic nearfield pressures produced by rectangular pistons, The Journal of the Acoustical Society of America 115 (2004) 1934-1941.

[4] B.T. Cox, P.C. Beard, Fast calculation of pulsed photoacoustic fields in fluids using k-space methods, The Journal of the Acoustical Society of America 117 (2005) 3616-3627.

[5] J.W.S. Rayleigh, The theory of sound, Dover publications, New York, 1945.

[6] Y.A. Kravtsov, Complex rays and complex caustics, Radiophysics and Quantum Electronics 10 (1967) 719-730.

[7] G.A. Deschamps, Gaussian beam as a bundle of complex rays, Electronics Letters 7 (1971) 684-685.

[8] A.L. Cullen, P.K. Yu, Complex Source-Point Theory of the Electromagnetic Open Resonator, Proceedings of the Royal Society of London. Series A, Mathematical and Physical Sciences 366 (1979) 155-171.

[9] M. Couture, P.A. Belanger, From Gaussian beam to complex-source-point spherical wave, Physical Review A 24 (1981) 355-359.

[10] L.B. Felsen, Geometrical theory of diffraction, evanescent waves, complex rays and Gaussian beams, Geophysical Journal of the Royal Astronomical Society 79 (1984) 77-88.

[11] F.G. Mitri, High-order pseudo-gaussian scalar acoustical beams IEEE Transactions on Ultrasonics, Ferroelectrics and Frequency Control 61 (2014) 191-196.

[12] F.G. Mitri, Quasi-Gaussian electromagnetic beams, Physical Review A 87 (2013) 035804.

[13] F.G. Mitri, Cylindrical quasi-Gaussian beams, Optics Letters 38 (2013) 4727-4730.

[14] T.D. Rossing, (Ed.), Handbook of Acoustics, Springer, New York, 2007.

[15] M. Abramowitz, I.A. Stegun, Handbook of Mathematical Functions: with Formulas, Graphs, and Mathematical Tables, Dover Publications, 1965.

[16] O.A. Sapozhnikov, An exact solution to the Helmholtz equation for a quasi-Gaussian beam in the form of a superposition of two sources and sinks with complex coordinates, Acoustical Physics 58 (2012) 41-47.

[17] G.N. Watson, A Treatise on the Theory of Bessel Functions, Cambridge University Press, London, 1922.

[18] J.D. Alemar, P.P. Delsanto, E. Rosario, A. Nagl, H. Uberall, Spectral-analysis of the scattering of acoustic-waves from a fluid cylinder .1. Denser fluid loading, Acustica 61 (1986) 1-6.

[19] j.d. Alemar, p.p. Delsanto, e. Rosario, a. Nagl, h. Uberall, Spectral-analysis of the scattering of acoustic-waves from a fluid cylinder .2. Denser fluid inside, Acustica 61 (1986) 7-13.

[20] j.d. Alemar, p.p. Delsanto, e. Rosario, a. Nagl, h. Uberall, Spectral-analysis of the scattering of acoustic-waves from a fluid cylinder .3. Solution of the inverse scattering problem, Acustica 61 (1986) 14-20.

[21] L.S. Schuetz, W.G. Neubauer, Acoustic reflection from cylinders---nonabsorbing and absorbing, The Journal of the Acoustical Society of America 62 (1977) 513-517.

[22] J.P. Lee, J.H. Song, M.S. Choi, The effects of material attenuation on acoustic resonance scattering from cylindrical tubes, Ultrasonics 34 (1996) 737 745.

[23] F.G. Mitri, Z.E.A. Fellah, J.Y. Chapelon, Acoustic backscattering form function of absorbing cylinder targets (L), Journal of the Acoustical Society of America 115 (2004) 1411-1413.

[24] F.G. Mitri, Radiation force acting on an absorbing cylinder placed in an incident plane progressive acoustic field, Journal of Sound and Vibration 284 (2005) 494-502.

[25] F.G. Mitri, Z.E.A. Fellah, Acoustic radiation force on coated cylinders in plane progressive waves, Journal of Sound and Vibration 308 (2007) 190-200.

[26] F.G. Mitri, Z.E.A. Fellah, Theoretical calculation of the acoustic radiation force on layered cylinders in a plane standing wave-comparison of near- and far-field solutions, Journal of Physics a-Mathematical and General 39 (2006) 6085-6096.

[27] J.D. Murphy, E.D. Breitenbach, H. Uberall, Resonance scattering of acoustic waves from cylindrical shells, Journal of the Acoustical Society of America 64 (1978) 677-683.

[28] T. Hasegawa, Y. Hino, A. Annou, H. Noda, M. Kato, N. Inoue, Acoustic radiation pressure acting on spherical and cylindrical shells, Journal of the Acoustical Society of America 93 (1993) 154-161.

[29] F.G. Mitri, Acoustic radiation force on cylindrical shells in a plane standing wave, Journal of Physics a-Mathematical and General 38 (2005) $9395-$ 9404.

[30] G.C. Gaunaurd, Sonar cross section of a coated hollow cylinder in water, Journal of the Acoustical Society of America 61 (1977) $360-368$.

[31] F.G. Mitri, Acoustic radiation force due to incident plane-progressive waves on coated cylindrical shells immersed in ideal compressible fluids, Wave Motion 43 (2006) 445-457.

[32] J.J. Faran, Sound scattering by solid cylinders and spheres, Journal of the Acoustical Society of America 23 (1951) 405-418.

[33] F.G. Mitri, Theoretical calculation of the acoustic radiation force acting on elastic and viscoelastic cylinders placed in a plane standing or quasistanding wave field, European Physical Journal B 44 (2005) 71-78.

[34] H.D. Dardy, J.A. Bucaro, L.S. Schuetz, L.R. Dragonette, Dynamic wide-bandwidth acoustic form-function determination, Journal of the Acoustical Society of America 62 (1977) 1373-1376.

[35] L. Flax, G.C. Gaunaurd, H. Uberall, Theory of Resonance Scattering - Ch. 3, in: W.P. Mason (Ed.), Physical Acoustics, Academic Press, New York, 1981, pp. 191-294.

[36] F.G. Mitri, Theoretical and experimental determination of the acoustic radiation force acting on an elastic cylinder in a plane progressive wave - farfield derivation approach, New Journal of Physics 8 (2006) 138.

[37] A.E. Siegman, Lasers, University Science Books, Mill Valley, CA, 1986.

[38] P.L. Marston, Quasi-Gaussian beam analytical basis and comparison with an alternative approach (L), The Journal of the Acoustical Society of America 130 (2011) 1091-1094.

[39] F.G. Mitri, Z.E.A. Fellah, Mechanism of the quasi-zero axial acoustic radiation force experienced by elastic and viscoelastic spheres in the field of a quasi-Gaussian beam and particle tweezing, Ultrasonics 54 (2014) 351-357.

[40] F.G. Mitri, Interaction of an acoustical Quasi-Gaussian beam with a rigid sphere: linear axial scattering, instantaneous force, and time-averaged radiation force, IEEE Transactions on Ultrasonics, Ferroelectrics and Frequency Control 59 (2012) 2347-2351. 
[41] M. Azarpeyvand, M. Azarpeyvand, Acoustic radiation force on a rigid cylinder in a focused Gaussian beam, Journal of Sound and Vibration 332 (2013) 2338-2349.

[42] X. Chen, R.E. Apfel, Radiation force on a spherical object in an axisymmetric wave field and its application to the calibration of high-frequency transducers, Journal of the Acoustical Society of America 99 (1996) 713-724.

[43] X. Zhang, G. Zhang, Acoustic Radiation Force of a Gaussian Beam Incident on Spherical Particles in Water, Ultrasound in Medicine \& Biology 38 (2012) 2007-2017.

[44] P.L. Marston, Axial radiation force of a Bessel beam on a sphere and direction reversal of the force, Journal of the Acoustical Society of America 120 (2006) 3518-3524.

[45] F.G. Mitri, Langevin Acoustic Radiation Force of a High-Order Bessel Beam on a Rigid Sphere, Ieee Transactions on Ultrasonics Ferroelectrics and Frequency Control 56 (2009) 1059-1064.

[46] F.G. Mitri, Negative axial radiation force on a fluid and elastic spheres illuminated by a high-order Bessel beam of progressive waves, Journal of Physics a-Mathematical and Theoretical 42 (2009) 245202.

[47] L.K. Zhang, P.L. Marston, Geometrical interpretation of negative radiation forces of acoustical Bessel beams on spheres, Physical Review E 84 (2011) 035601 .

[48] F.G. Mitri, Axial time-averaged acoustic radiation force on a cylinder in a nonviscous fluid revisited, Ultrasonics 50 (2010) $620-627$.

[49] T. Hasegawa, K. Saka, N. Inoue, K. Matsuzawa, Acoustic radiation force experienced by a solid elastic cylinder in a plane progressive sound field., Journal of the Acoustical Society of America 83 (1988) 1770-1775.

[50] W. Wei, D.B. Thiessen, P.L. Marston, Acoustic radiation force on a compressible cylinder in a standing wave, Journal of the Acoustical Society of America 116 (2004) 201-208.

[51] F.G. Mitri, Z.E.A. Fellah, Axial acoustic radiation force of progressive cylindrical diverging waves on a rigid and a soft cylinder immersed in an ideal compressible fluid, Ultrasonics 51 (2011) 523-526.

[52] F.G. Mitri, Erratum: Axial time-averaged acoustic radiation force on a cylinder in a nonviscous fluid revisited (vol 50, pg 620, 2010), Ultrasonics 51 (2011) 645 . 


\section{FIGURE CAPTIONS}

Fig.1. (Colour online) Geometry of the problem displaying a cross section of a cylinder centered on a coordinates system $(x O y)$. The parameter $r$ is the radial distance from the center of the coordinates system to an observation point $M, \theta$ is the polar angle in the $(x, y)$ cross-sectional plane where $x$ denotes the direction of wave propagation, and $a$ is the cylinder's radius. The other parameters are defined in the text.

Fig. 2. (Colour online) The plots for the magnitude of the velocity potential field of a cylindrical quasiGaussian beam for different values of the dimensionless waist of the beam $k w_{0}$. The wave number for the computations is $k=25 \times 10^{3} \mathrm{~m}^{-1}$, and the units along the axes are in $\mathrm{mm}$.

Fig. 3. (Colour online) The plots for the amplitude of the coefficient $\Lambda_{n}\left(k x_{R}\right)$ given by Eq.(3) vs. the partial-wave number $n$ for various values of the dimensionless waist number $k w_{0}$ noted on the figure.

Fig. 4. (Colour online) The plots for the magnitude of the backscattering form-function for $k w_{0}=1$ (solid line) and $k w_{0}=25$ (dashed line). The function $\left|f_{\infty}(k a, \theta=\pi)\right|$ approaches zero at $k a \sim 6.9$ for $k w_{0}$ $=1$.

Fig. 5. (Colour online) The same as in Fig. 4, but the plots correspond to the radiation force function $Y_{q G}$. There exists noticeable correlation between the zeros of the radiation force function $Y_{q G}$ for $k w_{0}=1$ with the minima of the magnitude of the backscattering form function. 